\title{
Procesos de incorporación sociocultural de la población inmigrante: asimilación y multiculturalidad
}

\author{
José Manuel Corpas Nogales ${ }^{1}$ \\ Universidad Nacional de Educación a Distancia
}

\section{Ensayo}

Material original autorizado para su primera publicación en el Journal de Ciencias Sociales, Revista Académica de la Facultad de Ciencias Sociales de la Universidad de Palermo.

\section{Recepción: 24-11-2020}

Aceptación: 11-06-2021

Resumen: En este ensayo se pone de relieve los diferentes enfoques y vertientes respecto a la incorporación de las personas inmigrantes. Concretamente, se analizan los conceptos y las ideas sociopolíticas y culturales en las que derivan términos como multiculturalidad, asimilación, pluralismo, integración o aculturación. Para ello, se realiza un análisis crítico de diferentes autores/as conversando entre los distintos términos y observando las consecuencias que tienen las diferentes perspectivas a la hora de estudiar la incorporación de las personas inmigrantes desde un continuum que va desde la asimilación total y forzada hasta el multiculturalismo/interculturalismo. Se trata pues de desentrañar la complejidad de las políticas socioculturales que se producen en las sociedades de acogida respecto a la asimilación/integración de las personas inmigrantes y de las múltiples relaciones que se pueden producir debido al encuentro de diferentes culturas. Para dicho análisis, en primer lugar, tenemos en cuenta la conceptualización, es decir qué entendemos por ciertos términos como asimilación, pluralismo, integración o multiculturalismo. Una vez aclarados los conceptos, nos interrogamos sobre la vuelta de ciertas políticas asimilacionistas que parecían haber desaparecido, pero que pueden haber regresado mediante formas más complejas y sutiles. A raíz de esta nueva asimilación, nos preguntamos sobre la heterogeneidad de dichos procesos en los diferentes grupos de inmigrantes para terminar cuestionándonos la gestión de la diversidad cultural en sus diferentes graduaciones, desde la asimilación hasta el multiculturalismo/interculturalismo.

Palabras clave: asimilación; multiculturalismo; integración social; aculturación.

\footnotetext{
${ }^{1}$ Doctor en Sociología, Universidad de Huelva. Licenciado en Antropología Social y Cultural y en Ciencias Políticas y Sociología, Universidad de Granada. Profesor Tutor UNED Centro asociado de Córdoba.

Correo electrónico: joscorpas@cordoba.uned.es
} 


\section{Processes of sociocultural incorporation of the immigrant population: assimilation and multiculturality}

Abstract: In this essay the different approaches and aspects regarding the incorporation of immigrants are highlighted. Specifically, the socio-political and cultural concepts and ideas are analyzed from which terms such as multiculturalism, assimilation, pluralism, integration or acculturation derive. For this, a critical analysis of different authors is carried out, talking between the different terms and observing the consequences that the different perspectives have when studying the incorporation of immigrants from a continuum that goes from total and forced assimilation to multiculturalism / interculturalism. It is therefore a question of unraveling the complexity of the sociocultural policies that are produced in host societies regarding the assimilation / integration of immigrants and the multiple relationships that can occur due to the encounter of different cultures. For this analysis, in the first place, we take into account conceptualization, that is, what we understand by certain terms such as assimilation, pluralism, integration or multiculturalism. Once the concepts have been clarified, we asked ourselves about the return of certain assimilationist policies that seemed to have disappeared, but which may have returned in more complex and subtle ways. As a result of this new assimilation, we wonder about the heterogeneity of these processes in the different immigrant groups, to end up questioning the management of cultural diversity in its different grades, from assimilation to multiculturalism / interculturalism.

Keywords: assimilation, multiculturalism, social integration, acculturation.

\section{Introducción}

El objetivo fundamental de este trabajo es analizar las diferentes actuaciones sociopolíticas que existen respecto a la convivencia que surge en aquellas sociedades donde la población inmigrante entra en contacto con las personas que ya habitan, desplegándose todo un bagaje cultural que puede colisionar, fusionarse o absorberse con la cultura predominante.

En este sentido los enfoques políticos y académicos que se han llevado a cabo mantienen una línea evolutiva sociohistórica que discurre desde políticas asimilacionistas donde la cultura de las personas inmigrantes queda progresivamente anulada incorporando poco a poco la cultura dominante de la sociedad receptora, hacia políticas más pluralistas, multiculturalistas e interculturales donde ambas culturas tienen sus aportaciones e interacciones desde un punto de vista más igualitario formando una fusión con aspectos destacados de las diferentes culturas. 
Sin embargo, esta línea evolutiva no está tan clara y se vuelve a plantear social y académicamente, tanto en Europa como en Norteamérica, el surgimiento de políticas actuales enfocadas de nuevo a la asimilación cultural de una forma más compleja, entremezclada, sutil y sinuosa.

Estas formas complejas de políticas socioculturales que se han producido y se están produciendo son el objeto de reflexión de este ensayo, para ello a continuación presentamos una argumentación teórica teniendo en cuenta obras clásicas en referencia a la asimilación y el multiculturalismo. Se desarrolla una conceptualización de los términos utilizados en materia de integración social respecto a la población inmigrante en las sociedades receptoras para debatir en torno a la posible vuelta de las políticas asimilacioncitas y la gestión de la diversidad cultural que se ha generado y se está produciendo a través de diferentes enfoques socioculturales.

\section{Conceptualización. Entre la asimilación y el multiculturalismo}

En primer lugar, para desarrollar este ensayo, nos debemos preguntar ¿qué entendemos por asimilación?, para ello, a continuación, vamos a intentar definir el término asimilación y los conceptos que se aproximan a dicha definición y que se encuentran en un itinerario entre la asimilación total y el multiculturalismo; teniendo en cuenta las aportaciones de los diferentes autores/as destacados para este fin.

Brubaker (2001) propone dos conceptos de asimilación, un concepto general y abstracto y otro específico y orgánico. La asimilación general y abstracta se refiriere a una asimilación hacia el proceso de hacerse iguales, mientras que el concepto específico y orgánico de asimilación se basa en un significado transitivo, partiendo de la idea de que para asimilar hay que convertir y señalando la importancia del resultado final y no del proceso; de esta forma la asimilación se desmarca de una categoría gradual. Además, dicho autor diferencia tres tipos de asimilación: la asimilación segmentada, la estructural y la espacial. La asimilación segmentada pone de manifiesto el éxito socioeconómico para los inmigrantes de segunda generación en barrios formados por minorías étnicas debido a que resisten a la asimilación en su medio juvenil circundante presentando a la vez una asimilación en el medio dominante. Por otro lado, la asimilación estructural tiene en cuenta la participación social de la población inmigrante en clubes o instituciones de la sociedad central a nivel de grupo primario, mientras que la espacial tiene en cuenta la suburbanización y la disminución de barrios étnicos (Brubaker, 2001).

Por su parte, Alba y Nee (1997) utilizan los términos aculturación y asimilación estructural entendiendo la aculturación como la adopción por parte del grupo minoritario de 
los patrones culturales de la sociedad de acogida. Y asimilación estructural como la entrada de las personas inmigrantes en grupos pequeños en dicha sociedad, como clubes, instituciones, asociaciones de ocio, etc. Gans (1997), además de conceptualizar la asimilación, presenta el término de retención étnica frente a un posible multiculturalismo. Por asimilación se refiere a la salida de los recién llegados de las asociaciones étnicas formales e informales y de otras instituciones sociales hacia los equivalentes no étnicos a los que puede acceder en esa misma sociedad de acogida. Mientras que aculturación sería la adopción por parte de los recién llegados de aspectos socioculturales procedentes de la sociedad de acogida, haciendo referencia a la retención étnica como un proceso por parte de los grupos de personas inmigrantes que evitan la aculturación y en su lugar mantienen sus lazos étnicos del lugar de procedencia.

Un término muy parecido a la asimilación es la aculturación. La aculturación hace referencia a la adopción por parte de los recién llegados de aspectos socioculturales procedentes de la sociedad de acogida, como podemos comprobar esta definición es muy parecida al concepto de asimilación, la diferencia está en que la aculturación se produce de forma voluntaria mientras que la asimilación se puede ejercer a través de mecanismos que anulan la voluntad del grupo. Además, la asimilación se entiende como la adaptación total a la nueva cultura, mientras que la aculturación sería el proceso de aprendizaje de esa nueva cultura, por lo tanto, dicho proceso puede acabar en asimilación o no. Por otro lado, se presenta el concepto de retención étnica que sería el proceso de resistencia a la asimilación, manteniendo y conjugando los aspectos socioculturales de origen con los del país de acogida.

También se propone en este apartado otros conceptos en relación a los procesos de incorporación social de las personas inmigrantes como son los aspectos de crisol de culturas, ensaladera, mosaico cultural y multiculturalismo catalizador, expuestos por Bauböck (2003). El autor, señala que la perspectiva asimilacionista se ha asociado con la imagen de crisol de culturas mientras que el multiculturalismo ha sido asociado con una ensaladera en la que cada ingrediente conserva su sabor distintivo y la forma, pero que se vuelve más interesante cuando se separan y se añaden los ingredientes. Por otro lado, se muestra el concepto mosaico cultural, recurriendo a la metáfora de piedras monocromáticas que crean la impresión de una imagen multicolor, al igual que las sociedades multiculturales contemporáneas.

En una versión más pluralista de la concepción de la incorporación de la población inmigrante en los países de acogida, nos encontramos con el término multiculturalismo. Glazer (1993) destacó, más que el concepto de multiculturalismo, el planteamiento basado en como el multiculturalismo ha surgido por la falta de asimilación de cierta población en Estados Unidos, observando la disminución de la actitud positiva con respecto a la 
asimilación, proponiendo que lo que alguna vez se consideró el sueño americano, ahora se ve con una luz negra y negativa.

El multiculturalismo se plantea, en un principio, como una forma de contacto entre personas con diferentes culturas, a partir de este encuentro surge la necesidad de gestionar la diversidad cultural. Por una parte, la asimilación da respuesta a esta gestión haciendo que la cultura de la sociedad emisora sea absorbida por la cultura de la sociedad de acogida, mientras que el multiculturalismo aboga por una relación más igualitaria entre las diferentes culturas, aportando ambas ciertos aspectos y relaciones socioculturales más recíprocas. En este sentido debemos exponer a continuación el concepto de integración, para ello hago referencia a Grillo (2007) que considera la integración como un proceso de inclusión y aceptación de los migrantes en las principales instituciones, las relaciones sociales y los estados de la sociedad receptora de larga duración. De esta forma, lo concibe no como un proceso de aplanamiento de la asimilación sino como igualdad de oportunidades, junto con la diversidad cultural, en una atmósfera de tolerancia mutua.

Según las ideas planteadas, podemos conceptualizar el término asimilación como un proceso mediante el cual las personas inmigrantes de diferentes culturas van dejando poco a poco sus referentes culturales del país del que proceden mientras que toman como estructura sociocultural de referencia los valores de la cultura del país de acogida. A su vez, este tipo de asimilación puede desarrollarse de diferentes formas dando lugar a varios tipos de asimilación. A continuación, hablaríamos de aculturación donde la persona inmigrante adopta muchos de los aspectos socioculturales de la sociedad de acogida, pero manteniendo ciertos lazos de unión con su cultura de origen. Siguiendo la línea del continuum, nos encontraríamos con la retención étnica entendida como un proceso por parte de los grupos de personas inmigrantes que evitan la aculturación y en su lugar mantienen sus lazos étnicos del lugar de procedencia. Y llegaríamos al multiculturalismo donde las relaciones entre las diferentes culturas se tienen en cuenta e incluso pueden llevar a provocar una hibridación de los diferentes aspectos socioculturales tanto de la cultura de origen como de la de acogida en diferentes grados estructurales. Dando un paso más, señalamos también el término interculturalismo entendido como la construcción de sociedades multiculturales, donde de forma más analítica y normativa se pretende realizar actuaciones que marquen relaciones "más allá de la tolerancia, la coexistencia y la igualdad formal entre culturas, para favorecer el respeto, la convivencia y la igualdad sustancial" (Cruz, 2013, p.71).

En definitiva, podemos observar los diferentes conceptos planteados desde un continuum que va desde la asimilación completa de las personas inmigrantes en la sociedad de acogida hasta un interculturalismo integrador, transitando por el término aculturación y por 
la retención étnica. Una vez expuestos los conceptos pasamos a dilucidar ciertas cuestiones en torno a dichos términos.

\section{Retorno de las políticas e ideas asimilacionistas heterogéneas en Estados}

\section{Unidos y Europa}

La siguiente pregunta que nos va a ayudar para aclarar el proceso de asimilación de las personas inmigrantes es ¿han vuelto las políticas asimilacionistas? En un sentido amplio de la historia podemos decir que se ha pasado de unas políticas asimilacionistas hacia unas políticas basadas en la multiculturaldiad o la retención étnica, pero existen diferentes autores que piensan que en la actualidad se está volviendo a dar políticas asimilacionistas; evidentemente con características diferentes a épocas anteriores y además revestidas de un aire moderno y conciliador, pero en el fondo siguen siendo políticas asimilacionistas. Un autor destacado que mantiene esta opinión es Brumaker (2001) que plantea que se está produciendo un giro hacia un discurso y un pensamiento social basado en el asimilacionismo, no como en la época anterior de los años ochenta, pero sí a través de un análisis más complejo y de una normativa más defendible que hace que el asimilacionismo sea más sutil, manteniendo la idea de que ha retornado dejando a un lado las posturas diferencialistas. Dicho autor, señala como ejemplos de retorno hacia la asimilación a tres países. El primero sería Francia, mediante una asimilación del discurso público, el segundo Alemania con una asimilación a través de políticas públicas y el tercero Estados Unidos donde la asimilación se produce debido a las perspectivas academicistas. Desde nuestro punto de vista, en España también se están volviendo a tomar perspectivas asimilacionistas, aún no desde el punto de vista político y académico, pero sí se están volviendo a producir un pensamiento colectivo, aún minoritario, que cuestiona todas las ideas interculturales y pluralistas a favor de unas políticas totalmente asimilacionistas, tomando ya parte del discurso público como sucede en Francia.

Por lo tanto, podemos responder a la pregunta planteada afirmando que se siguen generando políticas asimilacionistas, la pregunta que surge a continuación es ¿la asimilación que se produce en las personas inmigrantes es igual para todos los grupos de inmigrantes? La mayoría de las ideas aportadas por diferentes autores señalan que no, que la asimilación se está produciendo, pero a escalas diferentes en función de los grupos de inmigrantes. Por ejemplo, Glazer (1993) analiza la percepción que se ha tenido de las políticas asimilacionistas en respuesta a una sociedad cada vez más multicultural. Para ello, centra su trabajo en Estados Unidos analizando las políticas asimilacionistas en la población inmigrante, poniendo especial hincapié en la resistencia a la asimilación por parte de la población negra y los inmigrantes italianos. Los principales datos que muestra dicho autor, reflejan unos resultados 
diferentes en las políticas asimilacioncitas por parte de dichos grupos, puesto que los italianos tuvieron una transición algo suave hacia la cultura estadounidense, con las únicas barreras de las diferencias físicas y del idioma, sufriendo mucho menos conflictos y sorteando menos obstáculos que otros grupos culturales. Dichas políticas asimilacionistas ejercían como vehículo que transportaba a los italianos hacia la cultura estadounidense, sin embargo, tuvieron efectos enormemente negativos para la población afroamericana en los Estados Unidos.

Como podemos observar, existen diferencias pronunciadas entre los procesos de asimilación de los inmigrantes italianos y la población negra en Estados Unidos en función de variables como la religión, el idioma, las características fenotípicas, etc. En esta misma línea de pensamiento Alba y Nee (1997) argumentan que la teoría de la asimilación no ha perdido su utilidad y plantean que las políticas asimilacionistas se siguen produciendo, aunque de forma desigual. Mantienen la idea de una asimilación heterogénea, pasando desde una total asimilación hasta una asimilación nula. Dichos autores, exponen una sociedad que incluye a las diferentes clases sociales como la clase media y el proletariado, a personas de diferente color de piel, incluso a clases sociales por debajo de la clase obrera que se encuentran en situación de exclusión social. Para ello retoman datos en torno a la reconciliación de posturas contrarias en referencia a la asimilación, llegando a utilizar el marco teórico aislacionistas en situaciones muy heterogéneas.

Nos encontramos pues, ante una asimilación gradual y heterogénea que se produce de diferentes formas y dependiendo de cómo se lleven a cabo las diferentes posturas asimilacionistas pueden incluso llegar a provocar la exclusión social de la mayor parte de un grupo de personas inmigrantes, como sucede en muchos de los casos analizados tanto en Europa como en Estados Unidos. De esta forma, contestamos de forma rotunda a la pregunta realizada afirmando que la asimilación es una asimilación fragmentada o estratificada en los términos que señala Zhou (1997); el cual presenta la asimilación fragmentada como un marco teórico que explica los procesos de exclusión social que se producen en la segunda generación de inmigrantes contemporáneos en el país de acogida, produciéndose una asimilación estratificada.

\section{Procesos desiguales de asimilación, la asimilación fragmentada y}

\section{estratificada}

La pregunta que nos realizamos a continuación es ¿por qué se produce dicha asimilación fragmentada en algunos grupos de inmigrantes y por qué estos grupos son susceptibles de una movilidad descendente? Para contestar a esta pregunta debemos desentendernos de la asimilación clásica que propone que la asimilación es un proceso lógico 
y natural que se produce entre dos culturas diferentes en un mismo espacio. Con el tiempo ambas compartirán la cultura común del país de acogida a través de un proceso donde los patrones culturales del país de origen se abandonan progresivamente en favor de los de la sociedad receptora, de eta forma ambas culturas tienen una igualdad de oportunidades para la movilidad social ascendente en la estructura social del país de acogida. Es decir, según el asimilacionismo clásico se parte de una igualdad de oportunidades enfocadas en la meritocracia del sueño americano y de la supuesta movilidad social igualitaria planteada por el sistema económico neocapitalista. Sin embargo, el asimilacionismo estratificado plantea lo contrario, existe una movilidad descendiente en minorías étnicas por la exclusión socioeconómica de estos grupos debido a ciertas características que le son directamente atribuidas. Puede que tras varias generaciones desaparezcan las connotaciones negativas de estatus social y las oportunidades económicas basadas en la cultura y el idioma, pero se mantendrán otros factores de exclusión como la identificación del color de la piel, el idioma de origen y la religión, para determinar el nivel de aceptación de las minorías por parte del grupo dominante. De esta forma, la movilidad social ascendente de ciertos grupos de inmigrantes va a depender en gran parte del capital humano y financiero de sus familias, del contexto sociocultural al que pertenecen y de los patrones culturales aún híbridos entre la cultura del país de origen y el de acogida (Zhou, 1997).

Intentando dar respuesta a la cuestión planteada entendemos que las posibilidades de tener una movilidad ascendente en la sociedad de acogida son desiguales, pero por ello no hay que negar completamente las oportunidades de los grupos de inmigrantes. En este sentido, la teoría de la asimilación fragmentada plantea la asimilación de la población inmigrante en la sociedad de acogida desde diferentes posiciones socioeconómicas, concluyendo además que adoptar completamente la cultura del país de acogida no siempre puede ser una ventaja para ellos ni para sus hijos/as. Por lo cual, la movilidad social en los procesos de asimilación puede producirse hacia arriba o hacia abajo dependiendo fundamentalmente de la estructura sociocultural y económica de la familia y de los diferentes aspectos estratificadores de la sociedad de acogida. Es decir, la asimilación en la segunda generación de personas inmigrantes va a depender de la interacción entre factores estructurales y socioculturales.

Gans (2007) también analiza los procesos de asimilación y aculturación de las personas inmigrantes en función de la movilidad socioeconómica de éstos, teniendo como principal hipótesis que no existe una relación tan evidente entre movilidad socioeconómica y los procesos de asimilación y aculturación. La asimilación sería un proceso de adaptación de las personas inmigrantes a las condiciones socioculturales de la sociedad receptora, despojándose poco a poco de los aspectos socioculturales de la sociedad de origen, mientras que la movilidad económica se consideraría como el paso a un nivel superior o inferior del 
ingreso, la riqueza, la educación, la situación laboral y el nivel de vida. Por lo tanto, la idea que mantiene y que da respuesta a nuestra pregunta es que la asimilación por parte de las personas inmigrantes y la movilidad son procesos independientes, los inmigrantes pueden generar la asimilación sin tener una movilidad socioeconómica y viceversa.

Un claro ejemplo de las ideas expuestas sobre el proceso de asimilación estratificada se puede observar en el texto de Silberman et al. (2007) donde se analiza de forma comparativa los diferentes procesos de asimilación de algunas minorías étnicas en Estados Unidos y en Francia. Respecto a Francia se exponen los procesos de asimilación diferenciada de los grupos de segunda generación, sobre todo aquellos grupos de padres inmigrantes procedentes de países que fueron colonias francesas que se identifican con la religión musulmana. Los resultados respecto a estos grupos muestran un acceso desigual en tanto a la formación educativa, pero no se puede explicar la gran desigualdad que sufren en el mercado laboral únicamente teniendo en cuenta este factor, presentando además unos grandes niveles de desempleo respecto a los franceses nativos. Fundamentalmente, los grupos con unas características propias del proceso de exclusión social son los magrebíes de las antiguas colonias del norte de África, estas personas inmigrantes forman el grupo de segunda generación más grande de Francia y el más visible para los franceses nativos. Podemos hablar pues de una asimilación descendente y una instancia del valor del concepto de asimilación segmentada (Silberman et al. 2007). Además, como se ha señalado, la diferencia religiosa también es un factor importante a la hora de diferenciar entre el nativo francés y la segunda generación de magrebíes musulmanes.

En el caso de Francia la aculturación no se genera por la proximidad a una minoría étnica, más bien se produce porque se sigue manteniendo una relación con la sociedad de acogida, con una idea de rechazo ante el país colonizador, junto a una carencia de integración en el sistema educativo por los padres inmigrantes, proporcionando un modelo cultural alternativo a dicha segunda generación. Existe pues una oposición hacia la cultura hegemónica en los inmigrantes franceses que viven en las afueras de las ciudades en compartimentos estancos, creándose un círculo vicioso en torno a los procesos de inclusión y exclusión social. Silberman et al. (2007) concluyen que en Francia al igual que en Estados Unidos se producen mecanismos de asimilación fragmentada en la segunda generación de inmigrantes, sin embargo, los procesos que los generan en ambos países son procesos con diferentes características que deben ser estudiados y analizados en su contexto particular.

Como hemos podido observar en este ejemplo de asimilación fragmentada en Francia, unido al de los italianos o la población negra en Estados Unidos, la asimilación no se presenta de forma homogénea y en la mayor parte de los casos acaba provocando procesos de exclusión social y económica si los factores físicos, religiosos, lingüísticos y socioculturales 
difieren de los aspectos de la sociedad receptora. Además, desde un punto de vista de la sociedad de acogida también es fundamental las políticas que se lleven a cabo en el lugar de recepción, que no tiene por qué ser el país entero sino más bien políticas más locales, por ejemplo, políticas de gestión cultural teniendo como unidad de análisis la ciudad. En este sentido, Foner (2007), plantea como cuestión principal realizar un análisis de los procesos migratorios y de las relaciones multiculturales, teniendo en cuenta el enfoque de la ciudad como contexto. Para ello, toma como ejemplo el modo particular de multiculturalismo que se genera en la ciudad de Nueva York debido al contexto concreto de esta ciudad en cuanto a ciudad heterogénea, multicultural y con una buena percepción respecto a la acogida de la población inmigrante. La autora, destaca que en Nueva York existen unas políticas de acogida y de recepción de personas inmigrantes diferentes a otras ciudades de Estados Unidos, por ello es fundamental estudiar las relaciones multiculturales en los contextos urbanos determinados. Aunque Nueva York presenta unas políticas de etnicidad, sigue habiendo cierta discriminación y relaciones multiculturales basadas en compartimentos estancos, puesto que su estructura y política étnica está profundamente afectada por las leyes, las políticas y las instituciones nacionales.

Por lo tanto, es muy importante realizar un estudio focalizado en grupos concretos y en espacios concretos para recoger toda la heterogeneidad que se produce en los diferentes procesos de asimilación. Volviendo a poner a España como ejemplo, consideramos que hablar de unas políticas asimilacionistas a nivel de país resultaría demasiado desfocalizado puesto que poco tienen que ver las políticas asimilacionistas o multiculturalistas que se pueden llevar a cabo en regiones tan dispares como el País Vasco, Cataluña o Andalucía, o en la propia ciudad de Madrid dependiendo del mandato local.

\section{La gestión de la diversidad, entre la asimilación y el pluralismo}

Entramos, pues, en el debate de cómo gestionar la diversidad cultural, y en qué medida las políticas asimilacionistas ponen de relieve una incorporación social y cultural de ciertos grupos formados por personas inmigrantes. De ahí que la siguiente cuestión a debatir sea ¿la cohesión social de un país o de una sociedad se debe conseguir en base a políticas que gestionen la diversidad cultural o en base a la asimilación, en muchas ocasiones forzada? o más directamente ¿debemos pedirle a los recién llegados que se asimilen o quizás podemos generar una nueva sociedad con aspectos positivos de las diferentes culturas que ahora componen nuestros paisajes socioculturales?

En respuesta a estas preguntas, Bauböck (2003), mantiene la idea de que se siguen realizando políticas asimilacionistas y que además se intentan justificar mediante el sistema democrático como algo positivo e inevitable. Según dicho autor, a los/as inmigrantes se les 
pide que acepten un paquete de medidas que incluye no sólo la democracia, sino también la hegemonía de las culturas nacionales establecidas adoptando un compromiso de valores que algunos/as ciudadanos/as autóctonos/as ni siquiera cumplen.

Algunos/as autores/as opinan que la cohesión social en las sociedades debe ser construida sobre la base de diversidad en lugar de promover la similitud. Mientras que otros/as piensan que las democracias liberales han sido muy tolerantes con la inmigración, insistiendo en que la cohesión social debe producirse desde los valores e identidades compartidas. Los planteamientos de Bauböck (2003) se dirigen hacia la primera respuesta, según este autor la democracia incluye populistas xenófobos, de ahí que los valores democráticos no representen a toda la ciudadanía, añadiendo además que el enfoque asimilacionista hace que los inmigrantes olviden sus historias nacionales y adopten los valores socioculturales e históricos del país de acogida como si fuesen suyos.

Otra forma de entender la asimilación en los procesos de incorporación social de las personas inmigrantes es contraponer el asimilacionismo al multiculturalismo, por ello a continuación nos vamos a plantear ¿qué relación existe en los procesos de incorporación sociocultural de los grupos de personas inmigrantes en función de los procesos graduales entre la asimilación y el multuculturalismo? En este sentido, Gans (1997) considera que los análisis, de los/as investigadores/as sociales, entre asimilación y pluralismo se están acercando tanto que se pueden considerar desde un análisis conjunto. Destaca que ya no existe asimilación sino aculturación y que se ha pasado de un pluralismo a una retención étnica. Para ello, observa como éstos últimos conceptos comparten aspectos importantes de análisis llegando a "reconciliarse". Este análisis lo realiza mediante el proceso de cambio de la asimilación a la aculturación y del pluralismo a la retención étnica a través de la identidad cultural y del papel de los investigadores/as en función de si forman parte, o no, de la sociedad objeto de estudio. Según dicho autor, los asimilacionistas realmente se apoyan en la aculturación y los pluralistas han desarrollado una multiplicidad de significados que en realidad definen una retención étnica. Existen numerosas graduaciones entre dichos procesos puesto que la cultura étnica no se convierte en víctima de la aculturación, sino que se reconstruye o se reinventa todo el tiempo, por lo tanto, la identidad étnica puede ser compatible con la asimilación.

Las ideas planteadas nos dejan una mayor variedad de opciones a desarrollar entre el multiculturalismo y la asimilación, con ello me refiero al término de multiculturalismo catalizador de Bauböck (2003). Dicho autor, realiza esta metáfora porque un catalizador desencadena una reacción química que cambia la sustancia a la que se añade, de igual forma las relaciones multiculturales deben entenderse como un catalizador que pone en marcha un proceso de auto transformación de las identidades colectivas hacia una más pluralista. Sin 
embargo, que se tengan en cuenta políticas no asimilacionistas que prodiguen la multiculturalidad, no garantiza el hecho de que se produzca una integración sociocultural y económica de las personas inmigrantes en la sociedad de acogida, sino que puede suceder todo lo contrario. Como afirma Žižek (1998, p. 13): "el racismo posmoderno contemporáneo es el síntoma del capitalismo tardío multiculturalista, y echa luz sobre la contradicción propia del proyecto ideológico liberal-democrático". Con ello, el autor quiere decir que en el multiculturalismo existe una cultura dominante sobre otra que la construye desde aspectos negativos de la otredad. Es decir, construimos al "otro" con aspectos que consideramos que no son nuestros, de nuestra cultura. De esta manera, el multiculturalismo en la sociedad global y capitalista actual sirve para señalar la supuesta tolerancia multiculturalista en un mundo global donde se ha pasado de una colonización que destroza culturas a través del Estado nación a una hegemonía económica, social y cultural ejercida por las grandes corporaciones multinacionales recubiertas con un halo de multiculturalidad. Por lo cual, existe una idea problemática en torno al concepto de multiculturalismo que se impone desde una sociedad que aparenta el respeto hacia las culturas, pero desde una posición de privilegio frente a éstas, pudiendo despreciarlas en cualquier momento, siempre y cuando no perjudiquen al capitalismo como sistema mundial universal. Esta problemática multiculturalista es la que está produciendo una homogeneización sin precedentes en el mundo contemporáneo (Žižek, 1998).

Ante esta presencia cada vez más multicultural en las sociedades globalizadas se tienen que tomar decisiones que tengan en cuenta la gestión de la diversidad cultural, esta forma de gestionar la diversidad se enfrenta a dos polos opuestos entre aquellos/as que opinan que se debe fomentar una mayor integración de las personas con diferentes culturas, frente a los que piensan que se ha tolerado demasiado la diversidad cultural y por ello se están acrecentando los problemas en diferentes ciudades y países occidentales. Un ejemplo de esta problemática lo presenta Grillo (2007) haciendo referencia al caso de Europa en general y del Reino Unido en particular. Dicho autor describe la siguiente sucesión de hechos históricos en referencia a la gestión de la multiculturalidad en Europa diferenciando tres fases. La primera desde el siglo XIX hasta mitad deI XX donde el objetivo fundamental era intentar abolir la diferencia étnica con los Estados Nación que se asimilaban. La segunda fase, a partir de la segunda mitad del siglo XX hasta casi final de dicho siglo, donde se muestra el predominio de las normas de los Estados con más heterogeneidad y diversidad, realizándose una mayor integración dentro ya de un marco multicultural. Y la tercera fase, desde 1990 en adelante, en la cual se desarrollaron políticas de alteridad en exceso que nos ha llevado a un retorno a la asimilación, como plantea Brumaker (2001).

Hablar y escribir sobre multiculturalismo puede provocar la creación de un multiculturalismo imaginado frente a un multiculturalismo "real" que no está bien 
documentado ni definido. Por lo cual, los términos sobre el debate de la multiculturalidad como 'multiculturalismo', 'integración', 'diversidad' y 'diferencia' son conceptos en constante cambio que no se han terminado de conceptualizar creando incertidumbre en el debate. Por ello, debemos afrontar el análisis de la multiculturalidad desde un imaginario multisituado debido a los diferentes contextos terminológicos planteados anteriormente (Grillo, 2007). En definitiva, los planteamientos en torno a la asimilación como al multiculturalismo son objeto de crítica por los diferentes autores, señalando en casi todos que la situación de diversidad cultural se está gestionando de manera cada vez más asimilacionista, aunque ha habido un tiempo donde lo correcto era simular políticas de integración. Parece ser, que los problemas de una mala gestión de la diversidad cultural están haciendo que vuelvan a surgir conflictos en torno a las segundas y terceras generaciones de inmigrantes que aún no han encontrado su lugar en el país de acogida, como puede ser Francia, Reino Unido o los Estados Unidos.

\section{A modo de conclusión}

En primer lugar, vale destacar que, según lo expuesto, queda refutada la hipótesis que plantean diferentes autores/as clásicos cuando señalan que las políticas asimilacionistas han vuelto a producirse en Estados Unidos y en diferentes países europeos. Se está produciendo un retorno hacia nuevas políticas de asimilación, si es verdad que esta asimilación no es tan contundente como las que se llevaron a cabo en épocas anteriores, pero deja atrás el intento de construir una sociedad intercultural definida por la igualdad de oportunidades entre las personas pertenecientes a distintas culturas. Además, esta nueva asimilación ha regresado con una buena aceptación, una asimilación con una comprensión analítica, compleja y normativamente defendible, que ha producido un cambio en el propio concepto de asimilación. Ahora no se entiende la asimilación como una creencia global de lo inevitable sino, incluso, como un aspecto que puede ser positivo para la sociedad de acogida. Por lo tanto, lo que está sucediendo no es un retorno a los viejos tiempos de la asimilación arrogante, es un proceso que se centra en la asimilación para convertir a la ciudadanía en similar, un cambio de la asimilación transitiva a la intransitiva, que no se dirige hacia la persona individual sino hacia los grupos, que no se produce desde una unidad homogénea a otra, sino desde una heterogeneidad a otra y que promueve un concepto de asimilación holístico.

En este sentido podríamos hablar más que de procesos de asimilación de procesos de aculturación, aunque no debemos dejar de lado la teoría asimilacioncita como herramienta de análisis. Dicha teoría no ha perdido su utilidad, la asimilación está teniendo lugar, aunque de manera desigual, he aquí donde debemos poner el foco de la investigación analizando la asimilación segmentada y la heterogeneidad de dicho proceso teniendo en cuenta el contexto 
sociocultural particular de cada sociedad. De hecho, el análisis de los pluralistas o multiculturales también se deja influenciar por una cierta idea de superioridad de la cultura de la sociedad de acogida sobre la cultura de la sociedad emisora. Por lo cual, se ha producido un acercamiento de posturas entre la teoría analítica de los asimilacionistas y los pluralistas debido a que considera que los pluralistas han pasado a ser retencioncitas étnicos y la teoría de la asimilación ha derivado en aculturalismo.

Otra idea a concluir es que la asimilación que se está produciendo no se refiere a la asimilación clásica que abogaba por una igualdad de oportunidades entre los grupos de inmigrantes y el resto de la población, ahora se produce una estratificación que deja en desventaja a ciertos grupos de personas inmigrantes debido a la presencia de características socioculturales diferentes a las que presenta la sociedad de acogida. Además, este proceso sucede tanto en la primera generación de inmigrantes como en las siguientes, sobre todo si el grupo de personas inmigrantes lleva consigo factores socioculturales poco propicios a la aculturación como son el color de la piel, la religión o el idioma. Dichos factores unidos al capital humano y financiero de las familias de inmigrantes, junto con las estructuras políticas integradoras de la sociedad de acogida, van a condicionar el proceso de exclusión social de dicho colectivo.

Desde nuestro punto de vista, las políticas de asimilación no deben desarrollarse de manera forzada y debemos guiarnos, tanto para el análisis como para las políticas concretas, por planteamientos basados en procesos interculturales. Pienso que en ese continuum que va desde la asimilación total, la segmentada, las diferentes posturas de asimilación, la aculturación, la retención étnica, el multiculturalismo hasta que desemboca en la interculturalidad; debe prevalecer la idea de intentar conjugar de forma equitativa los valores socioculturales de las diferentes sociedades que se encuentran en un contexto concreto.

Actualmente el análisis de la incorporación sociocultural de las personas inmigrantes a una sociedad de acogida se produce desde formas muy heterogéneas, con graduaciones diferentes, creando una complejidad extrema, como para realizar un análisis fugaz donde todo se visualice desde la asimilación o el multiculturalismo. El investigador social debe realizar un estudio pormenorizado centrándose en el contexto concreto de cada caso, teniendo en cuenta las múltiples variables y las diferentes direcciones que estas variables pueden tomar. El posmodernismo y la globalización nos muestran esas múltiples direcciones entre lo local y global, no como una simple homogeneización de una cultura global y una asimilación completa de las culturas minoritarias; sino como unas relaciones que deben ser entendidas como superpuestas y globales y no como identidades predominantes. He aquí pues el reto del estudio de las relaciones interculturales en sociedades cada vez más volátiles y dinámicas donde la construcción y reconstrucción de las relaciones culturales pasan desde 
lo global a lo local, mediante fuerzas centrífugas y centrípetas a través de flujos multidireccionales que se plantean desde el extremo de la absoluta asimilación hasta el máximo intercultural.

\section{Referencias bibliográficas}

Alba, R. y Nee, V. (1997). Rethinking Assimilation Theory for a New Era of Immigration. International Migration Review, 31(4), 826-874. https://doi.org/10.2307/2547416

Bauböck, R. (2003). ¿Adiós al Multiculturalismo? Valores e identidades compartidos en las sociedades de inmigración. Revista de Occidente, (268), 45-61.

Brubaker, R. (2001). The return of assimilation? Changing perspectives on immigration and its sequels in France, Germany, and the United States. Ethnic and Racial Studies, 24(4), 531-548. https://doi.org/10.1080/01419870120049770

Cruz, E. (2013). Multiculturalismo e interculturalismo: una lectura comparada. Cuadernos Interculturales, 11(20), 45-76.

Foner, N. (2007). How exceptional is New York? Migration and multiculturalism in the empire city. Ethnic and Racial Studies, 30(6), 999-1023.

https://doi.org/10.1080/01419870701599440

Gans, H. J. (1997). Towards a Reconciliation of "Assimilation" and "Pluralism": The Interplay of Acculturation and Ethnic Retention. International Migration Review, 31(4), 875892. https://doi.org/10.1177/019791839703100404

Gans, H. (2007). Acculturation, assimilation and mobility. Ethnic and Racial Studies, 30(1), 152-164. https://doi.org/10.1080/01419870601006637

Glazer, N. (1993). Is Assimilation Dead? The ANNALS of the American Academy of Political and Social Science, 530(1), 122-136.

https://doi.org/10.1177/0002716293530001009

Grillo, R. (2007). An excess of alterity? Debating difference in a multicultural society. Ethnic and Racial Studies, 30(6), 979-998. https://doi.org/10.1080/01419870701599424

Silberman, R., Alba, R. y Fournier, I. (2007). Segmented assimilation in France? Discrimination in the labour market against the second generation. Ethnic and Racial Studies, 30(1), 1-27. https://doi.org/10.1080/01419870601006488

Zhou, M. (1997). Segmented Assimilation: issues, controversies, and recent research on the new second generation [Special Issue: Immigrant Adaptation and Native-Born 
Responses in the Making of Americans]. International Migration Review, 31(4), 975-1008. https://doi.org/10.2307/2547421

Žižek, S. (1998). Multiculturalismo, o la lógica cultural del capitalismo multinacional. En F. Jameson y S. Žižek, Estudios culturales. Reflexiones sobre el multiculturalismo (pp. 137-188). Paidós. 